\title{
Implementation of a digital evaluation platform to analyze bifurcation based nonlinear amplifiers
}

\author{
Sven Feldkord, Marco Reit, and Wolfgang Mathis \\ Institute of Theoretical Electrical Engineering, Leibniz Universität Hannover, Appelstraße 9A, 30167 Hannover, Germany \\ Correspondence to: Marco Reit (reit@ tet.uni-hannover.de)
}

Received: 15 January 2016 - Accepted: 11 March 2016 - Published: 28 September 2016

\begin{abstract}
Recently, nonlinear amplifiers based on the supercritical Andronov-Hopf bifurcation have become a focus of attention, especially in the modeling of the mammalian hearing organ. In general, to gain deeper insights in the inputoutput behavior, the analysis of bifurcation based amplifiers requires a flexible framework to exchange equations and adjust certain parameters. A DSP implementation is presented which is capable to analyze various amplifier systems. Amplifiers based on the Andronov-Hopf and Neimark-Sacker bifurcations are implemented and compared exemplarily. It is shown that the Neimark-Sacker system remarkably outperforms the Andronov-Hopf amplifier regarding the CPU usage. Nevertheless, both show a similar input-output behavior over a wide parameter range. Combined with an USBbased control interface connected to a PC, the digital framework provides a powerful instrument to analyze bifurcation based amplifiers.
\end{abstract}

\section{Introduction}

It is known for decades, that the mammalian hearing process involves an active amplification within the cochlea (Gold, 1948; Kemp, 1978). The amplification characteristic turned out to be similar to a system near the onset of an AndronovHopf bifurcation (Geisler, 1998; Eguíluz et al., 2000). A further work of Stoop et al. (2005) proposed a cochlea model that connects consecutive Hopf-type amplifiers with logarithmically decreasing characteristic frequencies. On this basis, an analog model was realized with discrete electronic elements (Stoop et al., 2005) and expanded to more than 20 cochlea sections (Stoop et al., 2007) to cover a wide range of frequencies. Each cochlea section includes a Hopf-type amplifier. The analog prototype reproduces the high sensitivity and compressive nonlinearity as well as the two-tone suppression and combination-tone generation with high precision (Stoop et al., 2007; Martignoli and Stoop, 2010). Each of them describes a characteristic feature of the cochlea. Nonetheless, the prototype shows unfavorable behavior due to tolerances and parasitic effects of the discrete electronic elements. Also the noise level adds up for consecutive stages (Stoop et al., 2007). Furthermore, larger changes in the underlying differential equations to analyze variations of the bifurcation based amplifiers requires rewiring or even building up a new system.

To overcome these drawbacks Reit et al. (2012) proposed a time-discrete implementation on a digital signal processor (DSP). Based on this work, the actual contribution presents a new implementation with significantly improved performance, user friendly control software and a modular approach that allows analyzing a variety of different systems. As an example, a new digital amplifier, based on the Neimark-Sacker bifurcation, is opposed to the AndronovHopf amplifier.

\section{Andronov-Hopf and Neimark-Sacker bifurcation fundamentals}

Since the Andronov-Hopf and Neimark-Sacker bifurcations both have been widely described in literature (e.g. Kuznetsov, 2013), only a brief overview of the used forms is given in this section. For the supercritical Andronov-Hopf bifurcation, the truncated normal form with the excitation $a(t)$ is used, where $z \in \mathbb{C}$ is the system variable, $\mu \in \mathbb{R}$ the bifurcation parameter and $\omega_{0}$ the characteristic frequency

$\frac{\mathrm{d} z}{\mathrm{~d} t}=\omega_{0}\left[(\mu+\mathrm{i}) z-|z|^{2} z+a(t)\right]$.

Equation (1) describes a $\omega_{0}$-rescaled variation of the normal form of the Andronov-Hopf bifurcation (Stoop et al., 2007). 


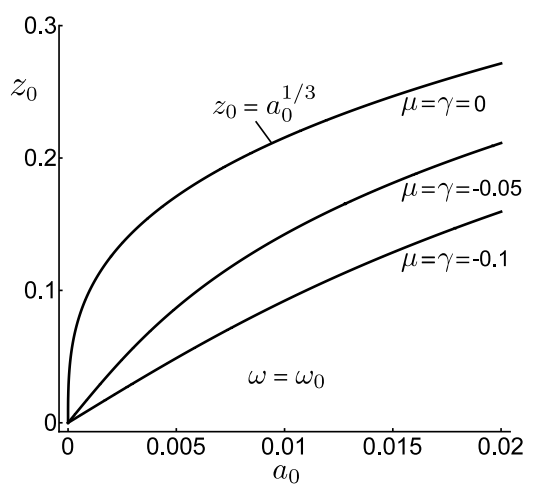

Figure 1. Identical input-output amplitude relations of the Andronov-Hopf and Neimark-Sacker systems for $\omega=\omega_{0}$.

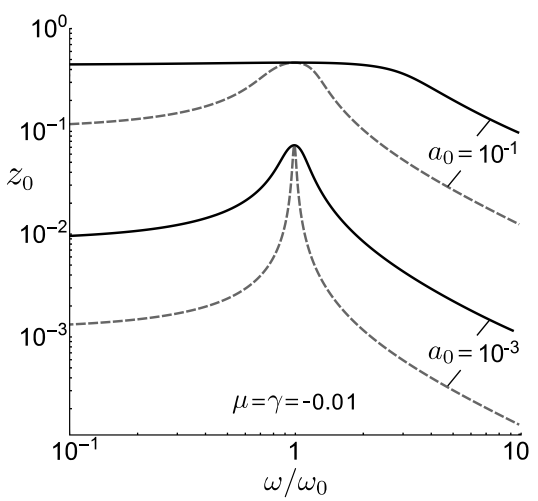

Figure 2. Amplitude response of the Andronov-Hopf system (dashed) and the Neimark-Sacker system (solid) for $\omega_{0}=1000$.

Without excitation, the system in Eq. (1) bifurcates at $\mu=0$ from a stable fixed point $\mu \leq 0$ to a stable limit cycle $\mu>0$. In order to operate as an amplifier, the driven system needs the parameter setting $\mu<0$. A special feature, as opposed to other nonlinear systems, is the mapping of a sinusoidal input signal $a_{0} \mathrm{e}^{i \omega t}$ to a sinusoidal output signal $z_{0} \mathrm{e}^{i(\omega t+\phi)}$ without harmonic distortions (Reit et al., 2013). This gives the possibility to describe the relation between the input amplitude $a_{0}$ and the output amplitude $z_{0}$ by the implicit equation

$a_{0}^{2}=z_{0}^{6}-2 \mu z_{0}^{4}+\left[\mu^{2}+\left(1-\frac{\omega}{\omega_{0}}\right)^{2}\right] z_{0}^{2}$.

The amplitude response of the Andronov-Hopf system in Eq. (1) is shown in Figs. 1 and 2 (dashed). Both show the nonlinear compression with strong amplification of weak signals near the characteristic frequency $\omega_{0}$ for small $\mu$-values. As can be noticed by Eq. (2), the input-output behavior depends on the $\omega / \omega_{0}$-ratio. For $\omega \rightarrow 0$ the system gets independent of $\omega_{0}$. Furthermore, small $\mu$-values $(\mu \rightarrow 0)$ and small input amplitudes $\left(a_{0} \ll 1\right)$ lead to $z_{0} \approx a_{0}$ for $\omega \rightarrow 0$ (cf. Fig. 2 (dashed)).
Opposed to the Andronov-Hopf bifurcation, the supercritical Neimark-Sacker bifurcation is a discrete-time system based on the nonlinear difference equation

$z \longmapsto \mathrm{e}^{i \theta} z\left(1+\gamma-|z|^{2}\right)+a(n)$.

As before, $z$ is the complex system variable. The bifurcation parameter is denoted by $\gamma$ and the supercritical bifurcation occurs, similar to the Andronov-Hopf system, at $\gamma=0$. For the investigations the parameter is set to $\gamma<0$. The excitation $a(n)=a_{0} \mathrm{e}^{i n \beta}$ is a sampled sine wave with the phase step $\beta$. The characteristic frequency has to be recalculated as a phase step $0<\theta<\pi$. When embedding the NeimarkSacker system in Eq. (3) into a time-continuous environment using $\mathrm{ADC}$ and $\mathrm{DAC}$, the relations

$\theta=2 \pi \frac{\omega_{0}}{\omega_{\mathrm{s}}}$ and $\beta=2 \pi \frac{\omega}{\omega_{\mathrm{s}}}$

where $\omega_{\mathrm{s}}$ is the sampling frequency, are obtained. Assuming a sinusoidal output signal, the input-output amplitude relation of Eq. (3) can be calculated to the implicit equation

$$
\begin{aligned}
a_{0}^{2}= & z_{0}^{2}\left(2+z_{0}^{4}-2 z_{0}^{2}(1+\gamma)+\gamma(2+\gamma)\right) \\
& +z_{0}^{2}\left(2\left(-1+z_{0}^{2}-\gamma\right) \cos (\beta-\theta)\right) .
\end{aligned}
$$

The amplitude response of the Neimark-Sacker system given by Eq. (5) is also shown in Figs. 1 and 2. It can be seen, that the input-output behavior is similar to that of the AndronovHopf bifurcation. For $\omega=\omega_{0}$ the amplitude relations are identical (cf. Fig. 1). In Fig. 2 the graphs (solid lines) show the same peak values as the Andronov-Hopf bifurcation (dashed lines) but the bandwidth is much wider and for $\omega \rightarrow 0$ the output amplitude is not approximately equal to the input amplitude. It can be shown, that for increasing values of $\omega_{0}$, the bandwidth gets more narrow and the curves of the Neimark-Sacker and Andronov-Hopf systems are increasingly converging. The term $\omega-\omega_{0}$ in the cos-function of Eq. (5) shows, that the input-output behavior of Eq. (3) depends directly on the $\omega_{0}$-value and not on the $\omega / \omega_{0}$-ratio, as the rescaled Andronov-Hopf system does.

\section{The digital implementation}

The most important goal for the digital implementation is to build a modular system that has the flexibility to exchange and adjust various parts of the system without the need to rewrite fundamental code. Furthermore, the implementation should provide the possibility to deactivate individual processing steps arbitrarily.

The processing steps themselves are based on the digital processing chain proposed by Reit et al. (2012) to build up a digital cochlea model. The improvement consists of a whole new framework for the digital signal processor ADSP-21369 from Analog Devices, written in assembler code. Thereby, 


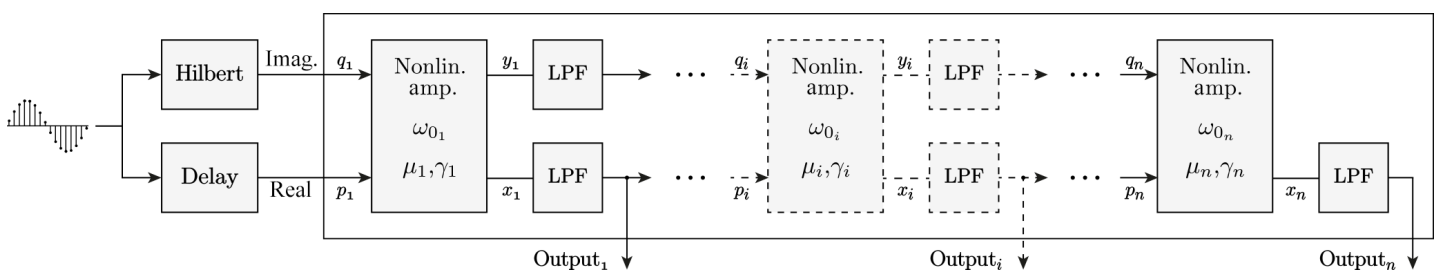

Figure 3. Modular processing chain

the focus is set on real-time performance, in-situ adjustment of parameters and the exchangeability of implemented differential equations. At first in the processing chain the incoming signals are sampled and highpass filtered to subdue DC parts. Afterwards, a Hilbert transform is computed to get an analytic input signal $a(t)=p(t)+\mathrm{j} q(t)$. This preprocessing block is followed by the main part of the chain, the consecutive bifurcation based amplifiers and lowpass filters (LPF) (cf. Fig. 3). For more informations regarding the structure see Reit et al. (2012). The processing block of the amplifier is implemented only once. For each link of the chain this unit is repetitively executed with the corresponding parameter values and input samples. The output is also represented by an analytic signal $z(t)=x(t)+\mathrm{j} y(t)$. To control the given parameters of the system, a graphical user interface (GUI) is designed for a PC, that communicates via USB-interface with the hardware.

The ADSP-21369 is a digital signal processor with Super Harvard Architecture (SHARC), an extension of the Harvard architecture by Analog Devices. The CPU contains a singleinstruction multiple-data computational architecture (SIMD) that enables the processor to perform up to 2.4 GFLOPS at $400 \mathrm{MHZ}$. The special structure of the DSP allows simultaneous writing and reading from a memory block, while processing data on a third block. The read and write operations are performed by serial port hardware blocks with chained DMA support that can communicate with the ADC and DAC via I2S protocol. The three block structure produces a delay of two times the blocklength. To get the total delay of the implementation, the system delay of the filters and amplifiers must be added.

Due to the high dynamic range of the system and the nonlinear compression, a number format with constant relative error instead of a constant absolute error seems feasible, so that the 40-bit IEEE floating point arithmetic is used, which is supported by the DSP. To solve the nonlinear differential equations of the bifurcation based amplifiers, a 4th-order Runge-Kutta method is chosen. This method appears to be a good choice related to stability, convergence and performance (Reit et al., 2012). Obviously, discrete systems given by an iterative map, such as the mentioned Neimark-Sacker system in Eq. (3), do not require an integration method since the mapping can be calculated once per time step.

Considering the equations are implemented by highly optimized assembler code, it is not possible for the user to en- ter arbitrary differential or difference equations by the GUI. In the first instance, it can be chosen between the nonlinear amplifiers based on the Andronov-Hopf and the NeimarkSacker bifurcation (see Sect. 2). A large number of consecutive sections can be calculated and for each of them, the characteristic frequency and the bifurcation parameter can be set individually. The coefficients for the three-stage biquad lowpass filter sections, that follows every amplifier stage optionally, can be adjusted as well. To analyze the specific behavior of one stage or one equation, any of the consecutive sections can be linked to the DAC of the output. Moreover, it is also possible to activate just one single processing section. To achieve full flexibility, it is also possible to deactivate the input highpass filter and the lowpass filters independently.

\section{Analysis}

The implemented framework was measured using an Audio Precision SYS-2522 measurement system. Different characteristic frequencies and bifurcation parameter values were tested with the Andronov-Hopf and the Neimark-Sacker systems, shown in Fig. 4. It can be seen that the measurements of the Andronov-Hopf system in Fig. 4a are qualitatively similar to the theoretical curves in Fig. 2 (dashed). However, noise issues occur for small input signals. For high characteristic frequencies, the input-output behavior of the Neimark-Sacker amplifier and the Andronov-Hopf amplifier is nearly indistinguishable (cf. Fig. 4a). For lower characteristic frequencies, larger deviations occur, which is obvious by comparing the appendant results in Fig. $4 \mathrm{~b}$ and c. This behavior can be explained by the rescaling of the Andronov-Hopf system which has not been found for the Neimark-Sacker system yet (see Sect. 2).

The performance of the implemented framework is outstanding. It is able to calculate twenty consecutive sections with about $44 \%$ CPU usage for the Andronov-Hopf and only $26 \%$ CPU usage for the Neimark-Sacker system. Table 1 shows a statistical profiling of the CPU usage for both systems with twenty sections where Solver means the system specific function for either integrating the differential equation or iterating the map. Hence, calculating the map of the Neimark-Sacker bifurcation is up to 4 times faster than calculating one time step using the 4th-order Runge-Kutta method. A further advantage of the Neimark-Sacker based 

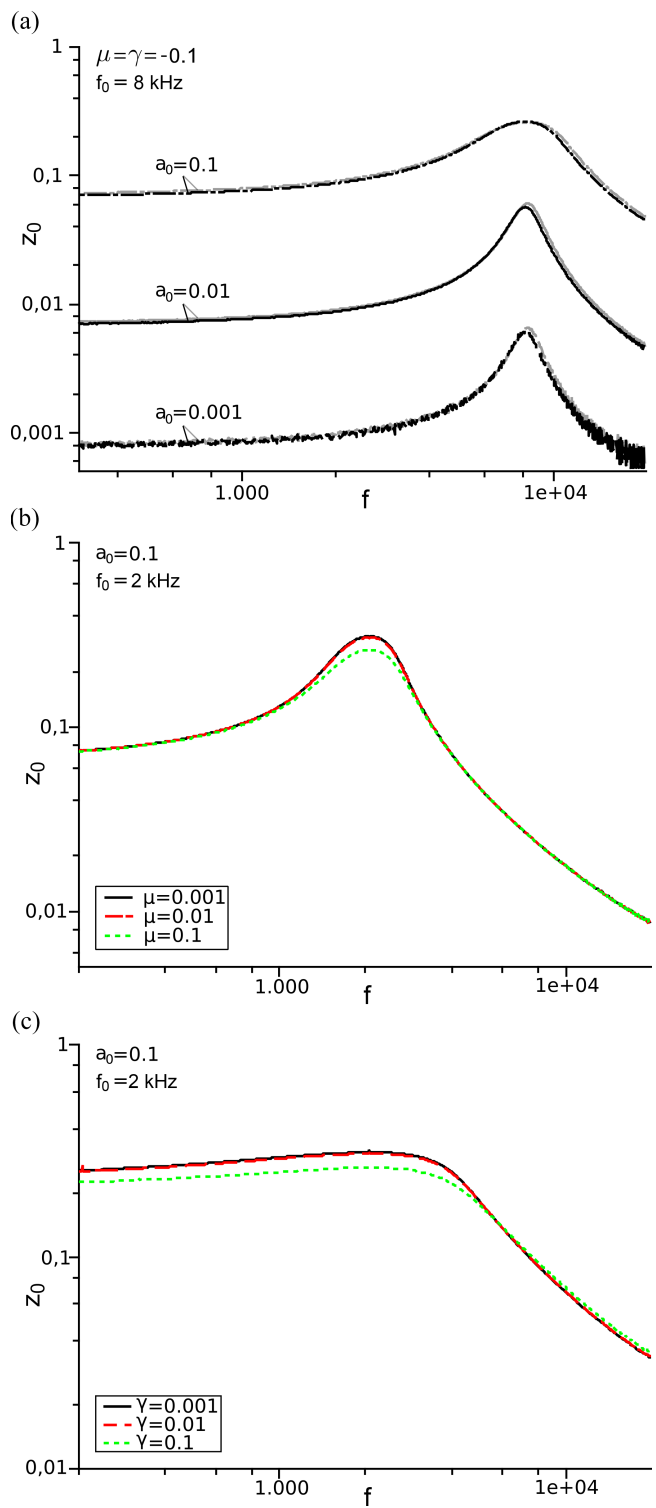

Figure 4. Amplitude responses versus frequency for $\omega_{0}=2 \pi f_{0}$; (a) Andronov-Hopf (grey) and Neimark-Sacker amplifier (black) for variation of $a_{0}$; (b) Andronov-Hopf amplifier for variation of $\mu$; (c) Neimark-Sacker amplifier for variation of $\gamma$.

Table 1. CPU usages for the Andronov-Hopf and Neimark-Sacker bifurcations.

\begin{tabular}{lcc}
\hline Activity & $\begin{array}{c}\text { CPU Usage (\%) } \\
\text { Andronov-Hopf }\end{array}$ & $\begin{array}{c}\text { CPU Usage (\%) } \\
\text { Neimark-Sacker }\end{array}$ \\
\hline Solver & $24.4 \%$ & $6.2 \%$ \\
\hline $\begin{array}{l}\text { Highpass, Hilbert, } \\
\text { Lowpass, Other }\end{array}$ & \multicolumn{2}{c}{$19.4 \%$} \\
\hline Total Usage & $43.8 \%$ & $25.6 \%$ \\
\hline
\end{tabular}

amplifier is the independency from an integration method. The Andronov-Hopf system can just be solved approximately and this underlies always integrator dependent damping and stability issues. The Neimark-Sacker system can be calculated precisely and has only to deal with the numerical precision which for the Andronov-Hopf system comes on top.

\section{Conclusions}

An implementation of a digital framework is introduced, that has the capability to analyze and compare various bifurcation based amplifiers, exemplarily shown by the Andronov-Hopf and Neimark-Sacker bifurcations. It is shown that the input-output behavior of the Neimark-Sacker system and the Andronov-Hopf system equals over a wide range of parameter sets for higher characteristic frequencies. Furthermore, the Neimark-Sacker system shows a considerable higher performance and robustness.

The publication of this article was funded by the open-access fund of Leibniz Universität Hannover.

Edited by: J. Anders

Reviewed by: two anonymous referees

\section{References}

Eguíluz, V. M., Ospeck, M., Choe, Y., Hudspeth, A., and Magnasco, M. O.: Essential Nonlinearities in Hearing, Phys. Rev. Lett., 84 5232-5235, doi:10.1103/PhysRevLett.84.5232, 2000.

Geisler, C. D.: From Sound to Synapse: Physiology of the Mammalian Ear, Oxford University Press, 1998.

Gold, T.: Hearing. II. The Physical Basis of the Action of the Cochlea, Proc. R. Soc. B, 135, 492-498, 1948.

Kemp, D. T.: Stimulated Acoustic Emissions from Within the $\mathrm{Hu}-$ man Auditory System, J. Acoust. Soc. Am., 64, 1386-1391, 1978.

Kuznetsov, Y. A.: Elements of Applied Bifurcation Theory, vol. 112, Springer Science \& Business Media, 2013.

Martignoli, S. and Stoop, R.: Local Cochlear Correlations of Perceived Pitch, Phys. Rev. Lett., 105, 048101, doi:10.1103/PhysRevLett.105.048101, 2010.

Reit, M., Mathis, W., and Stoop, R.: Time-Discrete Nonlinear Cochlea Model Implemented on DSP for Auditory Studies, in: Nonlinear Dynamics of Electronic Systems, Proceedings of NDES 2012, 1-4, 2012.

Reit, M., Mathis, W., and Stoop, R.: Analysis of Cascaded Canonical Dissipative Systems and LTI Filter Sections, Acta Technica, 59, 79-96, 2013.

Stoop, R., Kern, A., and van der Vyver, J.: How Ears Go Electronic, in: Proc. IEEE Conf. on Nonlinear Theory and its Applications (NOLTA), 2005.

Stoop, R., Jasa, T., Uwate, Y., and Martignoli, S.: From Hearing to Listening: Design and Properties of an Actively Tunable Electronic Hearing Sensor, Sensors, 7, 3287-3298, 2007. 